\title{
Stress waves propagation in solids under high-speed liquid impact
}

\author{
SHI Honghui ${ }^{1,2} \&$ J. E. Field ${ }^{3}$ \\ 1. State Key Laboratory of Nonlinear Mechanics, Institute of Mechanics, Chinese Academy of Sciences, \\ Beijing 100080, China; \\ 2. College of Mechanical Engineering and Automatic Control, Zhejiang University of Sciences, Xiasha \\ Higher Education Zone, Hangzhou 310018, China; \\ 3. Cavendish Laboratory, Cambridge University, Madingly Road, Cambridge CB3 OHE, UK \\ Correspondence should be addressed to Shi Honghui (email: hhshi@imech.ac.cn) \\ Received August 5, 2004
}

\begin{abstract}
An experiment of impact between $450 \mathrm{~m} / \mathrm{s}$ water jets and polymethylmethacrylate (PMMA) materials with complex surface geometry was conducted. The testing surfaces were a corner, step change, surface-breaking notch, inclined surface, etc. Stress waves propagation in the solid such as reflection, interference and diffraction was observed using polarized optics and an Imacon high speed camera (operating at both of $10^{6}$ and $5 \times 10^{5}$ framing rates per second, fps). A damage test by the impact of the side jetting of an $850 \mathrm{~m} / \mathrm{s}$ water jet was also carried out. It was found that stress waves propagation in solids depends not only on the surface geometry but also on the contact situation between liquid and solid. It was shown that the side jetting has sufficient damage potential although its head may consist of finer droplets. The results of this paper are useful to further analyze the dynamic stress state of solids under high-speed liquid impact.
\end{abstract}

Keywords: high-speed liquid/solid impact, stress waves, mechanical property of solid, high-speed photography.

DOI: 10.1360/03yw0223

The high-speed liquid/solid impact phenomenon is often seen in many technical fields such as, water jet cutting technology ${ }^{[1]}$, rain erosion of aviation vehicles ${ }^{[2]}$ and rotor blades erosion in large steam turbines ${ }^{[3]}$. Recently, China Aerodynamics Research Center reported experimental results of rain erosion of hypervelocity projectile ${ }^{[4]}$; Xi'an Jiaotong University reported numerical simulation of high-speed liquid drop impact on a solid surface ${ }^{[5]}$. In analyzing the mechanism of material's damage by liquid impact ${ }^{[6,7]}$, it is necessary to know the dynamic stress state in the solid so that it is also necessary to know stress waves propagation behaviors.

In accordance with elastic waves theory of solids ${ }^{[8]}$, when an impulse loading acts on a solid surface, a compression wave and a shear wave are generated in the solid bulk 
and on the surface, a Rayleigh surface wave is generated. When the compression wave propagates along the free surface, a shear wave called head wave appears, whose appearance is to offset the stress on the free surface caused by the compression wave. After the contact of a high-speed liquid drop (or jet) on a solid surface, side or lateral jetting whose velocity is a few times the impact velocity forms on the surface ${ }^{[3]}$. This fast side jetting causes a strong shear wave in solids and makes shear bands easily formed in the sub-layer of brittle materials ${ }^{[9]}$.

For rotor blades in large steam turbines, it is impossible to completely avoid erosion by water droplets impact. After working some period, the blades surface becomes more or less eroded but the blades still can be used for a quite long time. Therefore, water droplets impact on a rough surface rather than on a smooth surface. In ref. [10], Shi et al. studied the effect of the surface geometry on the impact pressure, fluid dynamics of liquid/solid impact and stress waves. This paper shows the experimental results of high-speed liquid on a corner, step change, surface-breaking notch and inclined surface. The key issue is the complicated process of stress wave's propagation. The relevant research work can be found in ultrasound detecting theory ${ }^{[11]}$ and earthquake waves prediction.

\section{Experimental device and method}

The experiment was done at the Cavendish Laboratory, Cambridge University, UK using the single impact jet testing apparatus (SIJT). Fig. 1 shows the schematic of the SIJT and the optical system of high speed photography. A lead slug was fired from a $5.59 \mathrm{~mm}$ in bore diameter high pressure nitrogen gas gun. The slug impacted on the liquid sealed in the nozzle by a neoprene. The impact generated high pressure in the nozzle and the pressure drove the liquid to flow through a $0.8 \mathrm{~mm}$ diameter nozzle exit to form a high speed liquid jet. The jet velocity could be adjusted by adjusting the pressure in the reservoir and using the solenoid valve. An Imacon high speed camera was used to ob-

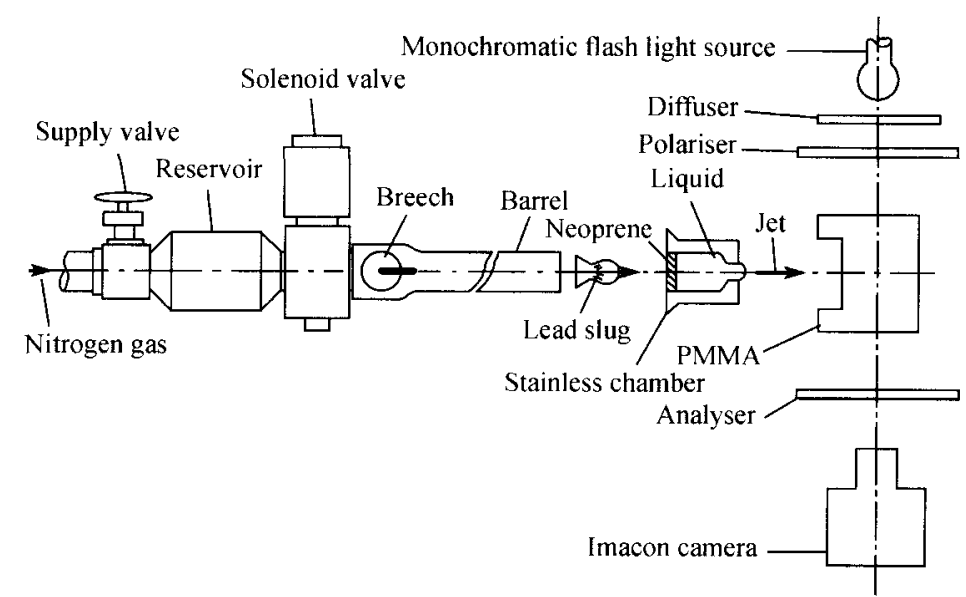

Fig. 1. Experimental device of high speed liquid/solid impact and high speed photography system. 
serve the impact process between water jets and PMMA blocks. A pair of linear polarizing plates were added in the optical system for viewing stress waves. According to different experimental conditions, framing rates at $10^{6}$ and $5 \times 10^{5}$ fps were used. Before the experiments, the jet velocity was calibrated using optical fiber measurement. The thickness of the testing PMMA material was all $25 \mathrm{~mm}$ and was made from ICI Glass Co. Ltd., UK.

\section{Experimental results}

\subsection{Reflection of stress waves at a corner}

Fig. 2 shows the impact sequences during a $450 \mathrm{~m} / \mathrm{s}$ water jet impact on a $90^{\circ}$ PMMA corner, which moves downwards from the upper part in the picture. In fig. 2(1), the jet head has just contacted the solid surface. Only after $1 \mu \mathrm{s}$, in fig. 2(2), stress waves appear in the solid; meanwhile, side jetting (labeled as $\mathrm{J}$ in the figure) forms and flows along the surface. The side jetting velocity has reached $1324 \mathrm{~m} / \mathrm{s}$.

As stress waves propagate in the sold bulk, the compression wave (marked as $\mathrm{C}$ in fig. 2(5)) and shear wave (marked as $S$ in fig. 2(9)) become distinguishable. The compression and shear waves reflect on the left free surface. Firstly, the compression reflection generates a reflected longitudinal wave (marked as $C^{\prime}$ in fig. 2(6)) and a reflected transverse wave (marked as $S^{\prime}$ in fig. 2(7)). The incident angle of wave $C$ equals the reflection angle of wave $C^{\prime}$. The definition of the incident and reflection angles is the angle between the normal to the free surface and the normal to the wave front. The reflection angle of wave $S^{\prime}$ can be calculated by Snell's law ${ }^{[12]}$. In fig. 2, the incident angle of wave $C^{\prime}$ is $65^{\circ}$, the reflection angle of wave $S^{\prime}$ is about $30^{\circ}$.

The reflection of a transverse wave on a free surface usually also generates a reflected transverse wave and a reflected longitudinal wave. However, if the wave's incident angle is greater than its critical angle, the reflected longitudinal wave will not appear but become a sort of surface wave ${ }^{[13]}$. The amplitude ratio between the incident and reflected transverse waves is -1 , so that these two waves are mode converted. The critical angle of PMMA is $25^{\circ}$. In fig. 2, the incident angle of wave $S$ is $68.5^{\circ}$, greater than the critical angle. As a result, only the reflected transverse wave $S_{t}$ is observable (fig. 2(9)). As mentioned above, waves $S_{t}$ and $S$ are of equal amplitude and opposite mode so that wave $S_{t}$ interferes with wave $S^{\prime}$. It is seen from fig. 2(7) to fig. 2(12) that the extensional line of wave $\mathrm{S}^{\prime}$ ends at the position of the $\mathrm{S}_{\mathrm{t}}$ wave front.

The Rayleigh surface wave passes through the corner tip between fig. 2(6) and (7). Then, part of it transmits over the corner (labeled $R_{t}$ in fig. 2(11)) and part of it is reflected back (labeled $R_{r}$ in fig. 2(12)). As shown in the figure, for a $90^{\circ}$ corner, the energy of wave $R_{t}$ is greater than that of wave $R_{r}$. 


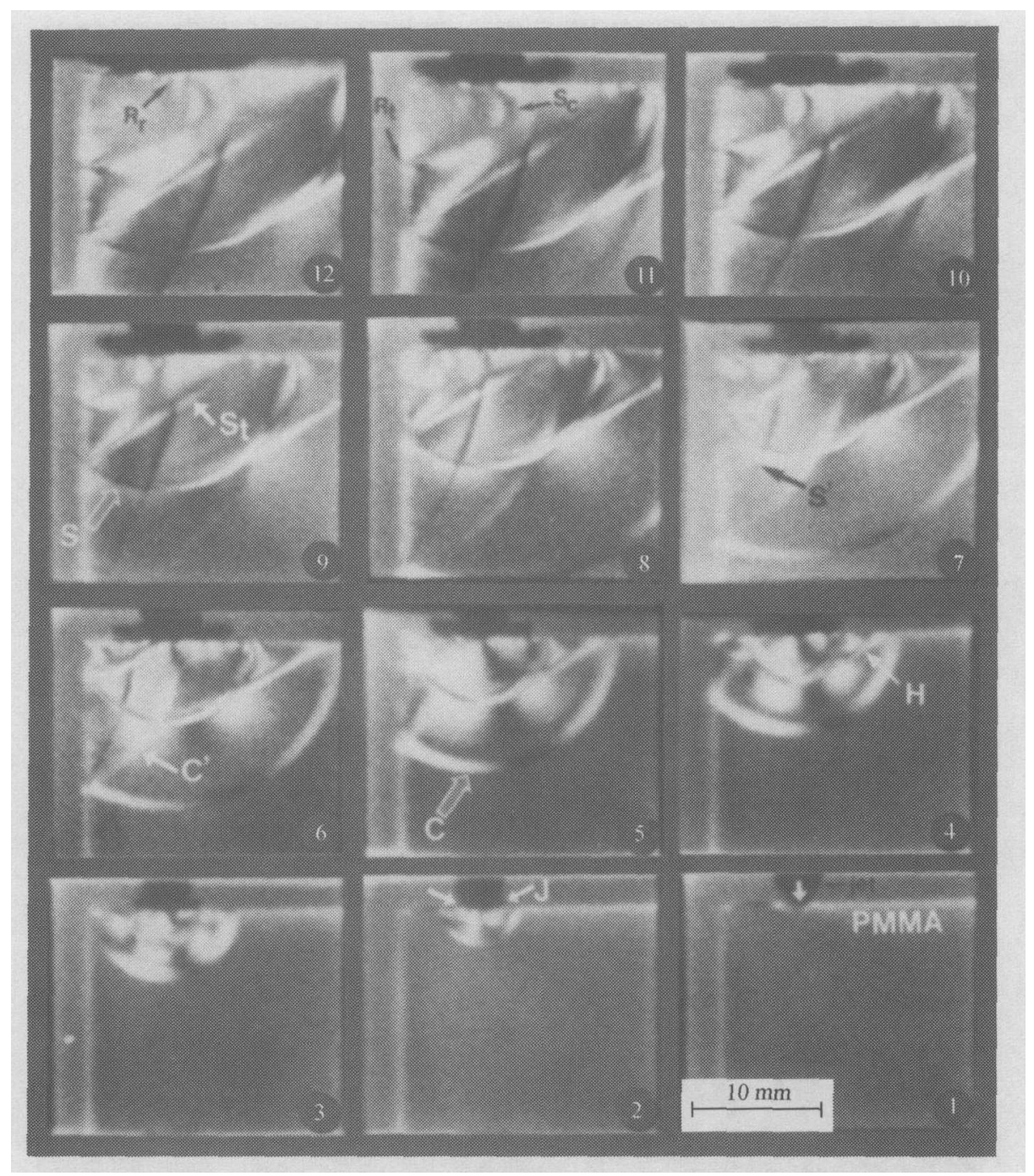

Fig. 2. Stress waves reflection at a $90^{\circ}$ corner. $450 \mathrm{~m} / \mathrm{s}$ water jet from a $0.8 \mathrm{~mm}$ diameter nozzle impacts on a PMMA block. In the figure, $\mathrm{C}$ is compression wave; $\mathrm{C}^{\prime}$ is the reflected compression wave of $\mathrm{C}$, it is a tensile wave; $\mathrm{S}^{\prime}$ is the reflected shear wave of $\mathrm{C}$; $\mathrm{S}$ is incident shear wave; $\mathrm{S}_{t}$ is the reflected transverse wave of $\mathrm{S}$; $\mathrm{S}_{c}$ is a transverse wave when $S$ is reflected back from the corner; $R_{t}$, transmitted surface wave through the corner; $R_{r}$, reflected surface wave from the corner; $\mathrm{H}$, head wave; J, side jetting. Note that wave $\mathrm{S}^{\prime}$ interferes with wave $\mathrm{S}_{\mathrm{t}}$. The reflected longitudinal wave of $\mathrm{S}$ becomes a surface wave, because its incident angle is greater than the critical angle. Interframe time $=1 \mu \mathrm{s}$.

\subsection{Diffraction of stress waves at surface discontinuity}

Fig. 3 shows high speed photographs of stress waves diffraction. On the testing PMMA surface, there is surface-breaking notch abcd which is $1.5 \mathrm{~mm}$ wide and $2 \mathrm{~mm}$ deep. On the right site of the notch, a $450 \mathrm{~m} / \mathrm{s}$ water impacts on the solid surface (the white arrow in fig. 3(A)). Stress waves caused by the impact propagate to the left and are 


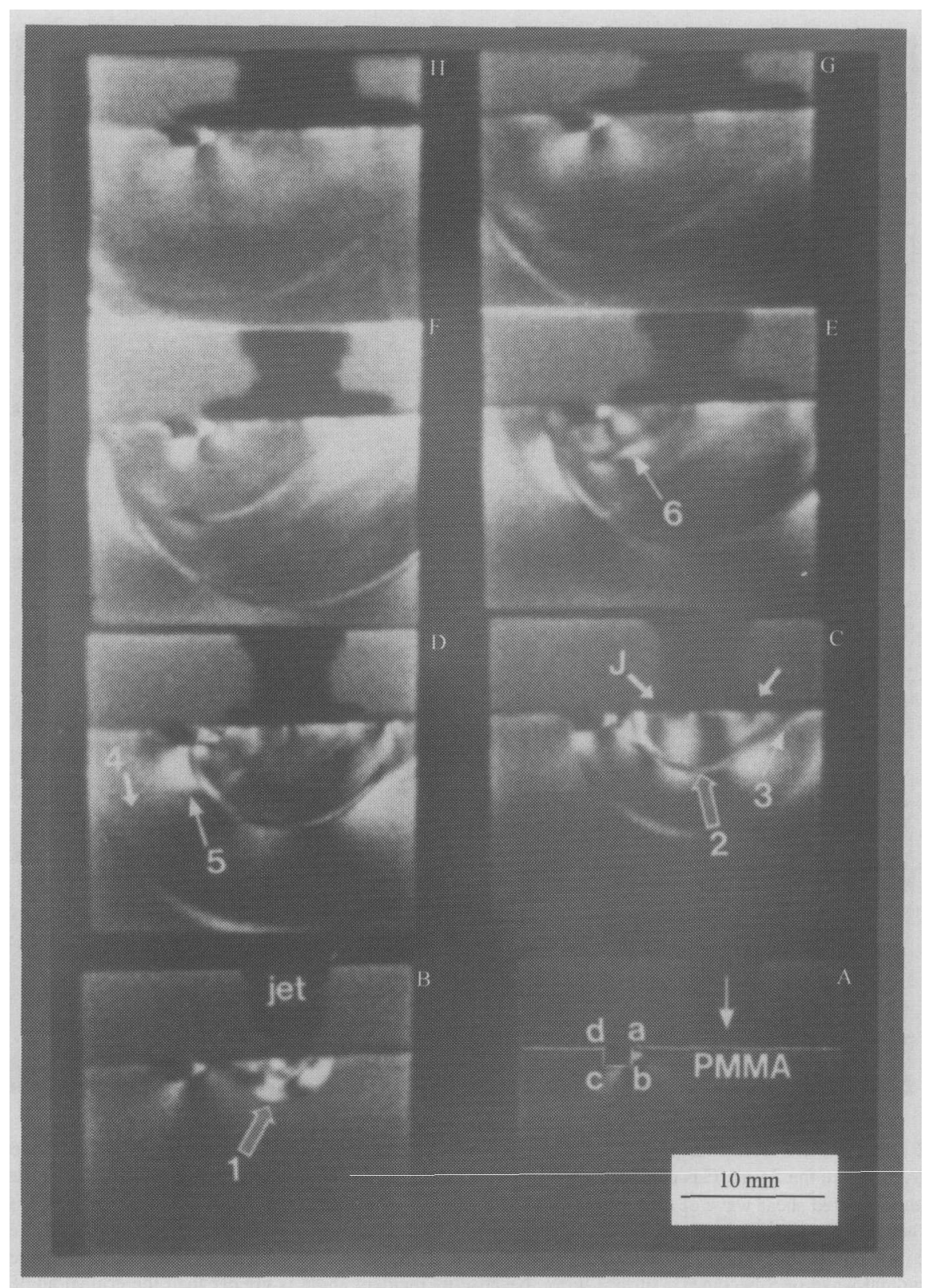

Fig. 3. Stress waves diffraction at a surface-breaking notch (abcd). $450 \mathrm{~m} / \mathrm{s}$ water jet impact on PMMA block. Point $b$ of the notch is the diffraction position. In the figure, 1 is compression wave; 2 is shear wave; 3 is head wave; 4 is diffracted longitudinal wave; 5 is diffracted head wave, its velocity is measured at $1396 \mathrm{~m} / \mathrm{s}$ so that it is a kind of shear wave; in frame E, 6 is the diffracted transverse wave of wave 2. In frame A, the white arrow shows the impact site. In frame $\mathrm{C}, \mathrm{J}$ is side jetting. Interframe time $=2 \mu \mathrm{s}$.

diffracted at point b. In fig. 3, 1 is incident compression wave, 2 is incident shear wave, 3 is head wave, 4 is diffracted longitudinal wave (because of the re-production of the photo, the wave front position becomes faint but it still can be found by carefully exam-

Copyright by Science in China Press 2004 
ining the circle line of the diffracted wave), 5 is diffracted head wave (its velocity is measured at $1396 \mathrm{~m} / \mathrm{s}$ so that it is a kind of shear wave), 6 (fig. 3(E)) is the diffracted transverse wave of the incident shear wave, $\mathrm{J}$ (fig. $3(\mathrm{C})$ ) is side jetting. In accordance with the theory of stress waves diffraction by a slit ${ }^{[13]}$, the diffraction of a compression wave generates both a longitudinal and transverse waves. However, no diffracted transverse wave of compression wave 1 can be found in fig. 3 , and this may be attributed to that its strength is too low or it may overlap with wave 5 (a kind of shear wave).

Fig. 4 shows high speed photographs of stress waves diffraction at a step change abcd. A $450 \mathrm{~m} / \mathrm{s}$ water jet impacts in the middle of the $10 \mathrm{~mm}$ wide step change bottom bc. The white arrow in fig. 4(A) shows the impact site. Stress waves appear in fig. 4(C). When stress waves propagate to two sides, diffractions occur at points b and c. The labels 1,2 and 3 in the figure mean compression, shear and head waves respectively. 4 and 4 are diffracted head waves on the left and right sides; 5 and $5^{\prime}$ are believed to be diffracted transverse waves of wave 2 at points b and c respectively. Actually, the stress fields at points b and c are very complicated since when high speed jetting impacts on side walls ad and dc, new stress waves will be generated.

From the high speed photographs shown in figs. 2-4, the velocity of the compression wave in PMMA is measured at $2536 \mathrm{~m} / \mathrm{s}$ and the velocity of the shear wave 1382 $\mathrm{m} / \mathrm{s}$.

\subsection{Liquid impact on notch and groove}

Fig. 5(1) shows the sequences of a $450 \mathrm{~m} / \mathrm{s}$ water jet impact on a $1.5 \mathrm{~mm}$ wide and $2 \mathrm{~mm}$ deep notch. The white arrow in the figure shows that the jet rightly impacts in the middle of the notch. In this case, the stress waves are somewhat different from those during the impact on a flat surface. The compression wave $\mathrm{C}$ becomes weak and is much weaker in the lateral direction; the shear wave $S$ is stronger and multiple shear waves appear. Theses waves are not generated by the high speed side jetting but generated by liquid extruding on the notch walls. This experiment confirms that a surface geometry change causes variation of liquid/solid contact mechanics. This can be further confirmed by the following two examples.

Fig. 6 shows another case of the jet impact on the notch. The white arrow in fig. 6(1) indicates that the jet impacts on the right corner of the notch. In fig. 6(2), stress waves start to propagate into the solid from the right upper corner. It is seen from fig. 6(4) that this kind of impact causes asymmetric shear wave S and head wave $\mathrm{H}$. After the jet is in contact with the solid wall, the right part of liquid forms a side jetting to flow along the wall surface (labeled $\mathrm{J}$ in fig. 6(3)). The velocity of $\mathrm{J}$ is about $800 \mathrm{~m} / \mathrm{s}$. The left part of liquid impacts on the notch bottom and then is rebounded back to form a jet J' moving towards upper-left direction. The velocity of the J' is about $625 \mathrm{~m} / \mathrm{s}$. The liquid impact on the notch bottom generates secondary compression and shear waves (labeled $C^{\prime}$ and $S^{\prime}$ in fig. 6(7) and 6(8)). 


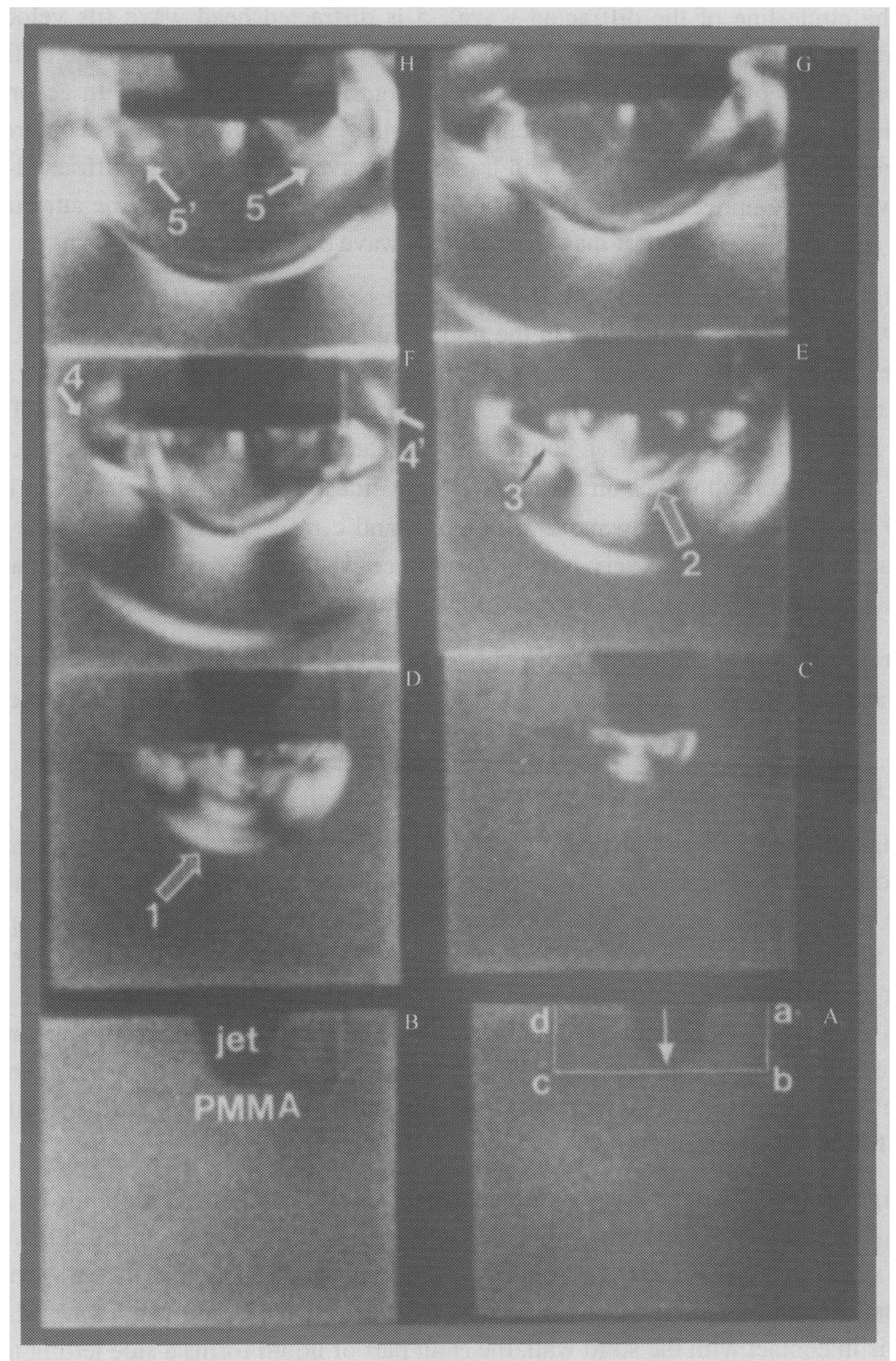

Fig. 4. Stress waves diffraction at a step change (abcd). $450 \mathrm{~m} / \mathrm{s}$ water jet impact on PMMA block. Points b and c of the step change are diffraction positions. The width of the step change is $10 \mathrm{~mm}$. In the figure, 1,2 and 3 are compression, shear and head waves respectively; 4 and $4^{\prime}$ are diffracted head waves at points $\mathrm{c}$ and b respectively; 5 and $5^{\prime}$ are diffracted transverse waves. Interframe time $=1 \mu$ s.

Fig. 7 shows high speed photographs of the jet impact on the bottom of a $90^{\circ} \mathrm{V}$ shape groove. In this case, the compression wave is weak (labeled C in fig. 7(2)) and

Copyright by Science in China Press 2004 


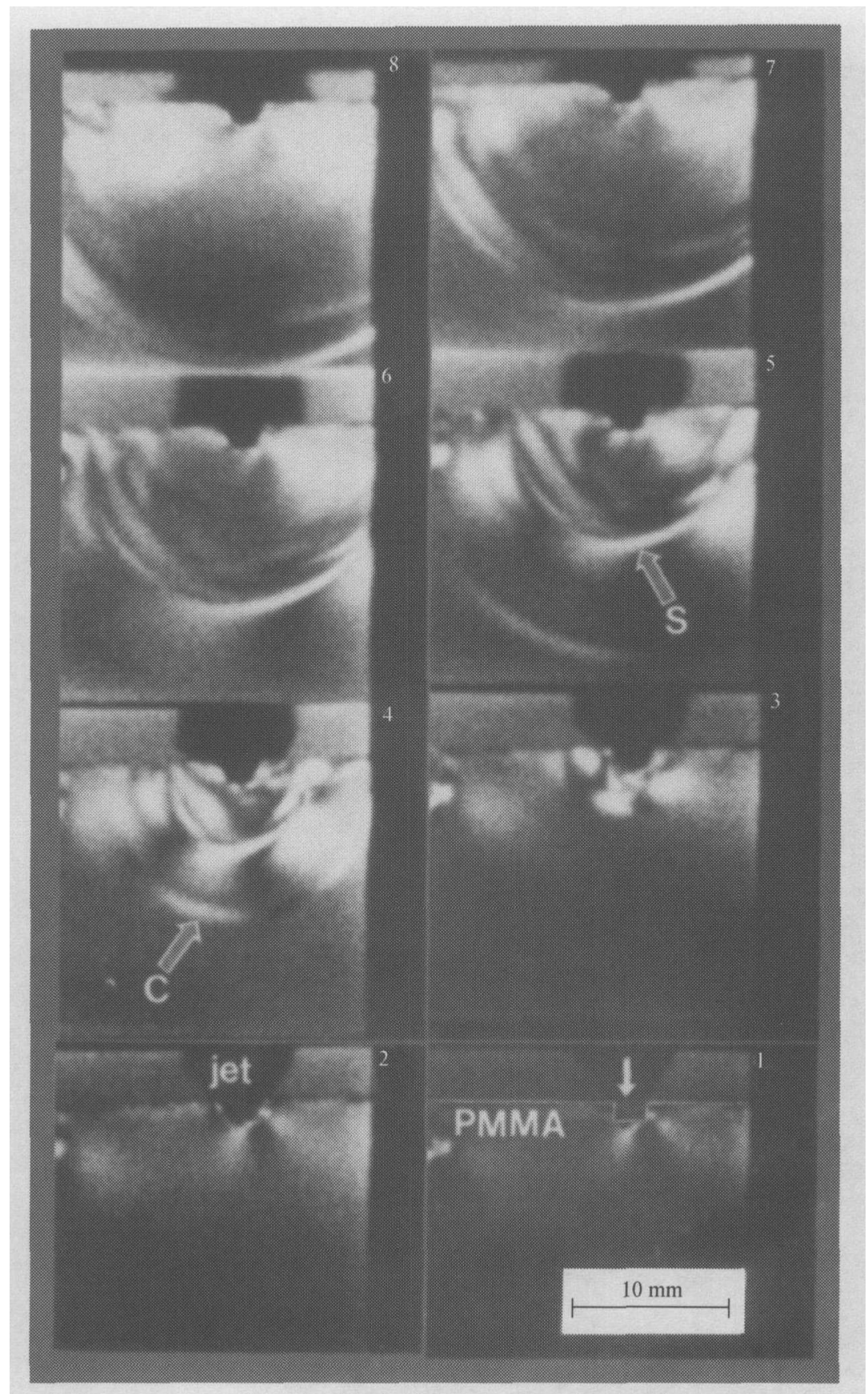

Fig. 5. High speed photographs of a $450 \mathrm{~m} / \mathrm{s}$ water jet normally impact on a PMMA notch. The white arrow in frame 1 shows the impact site. $C$ and $S$ are compression and shear waves respectively. Interframe time $=2 \mu \mathrm{s}$.

shear wave is strong (labeled $\mathrm{S}$ in fig. 7(3), in fig. 7(5) $\mathrm{S}_{\mathrm{t}}$ is reflected shear wave). The reason for this is that the jet head first contacts the left and right inclined surfaces of the 


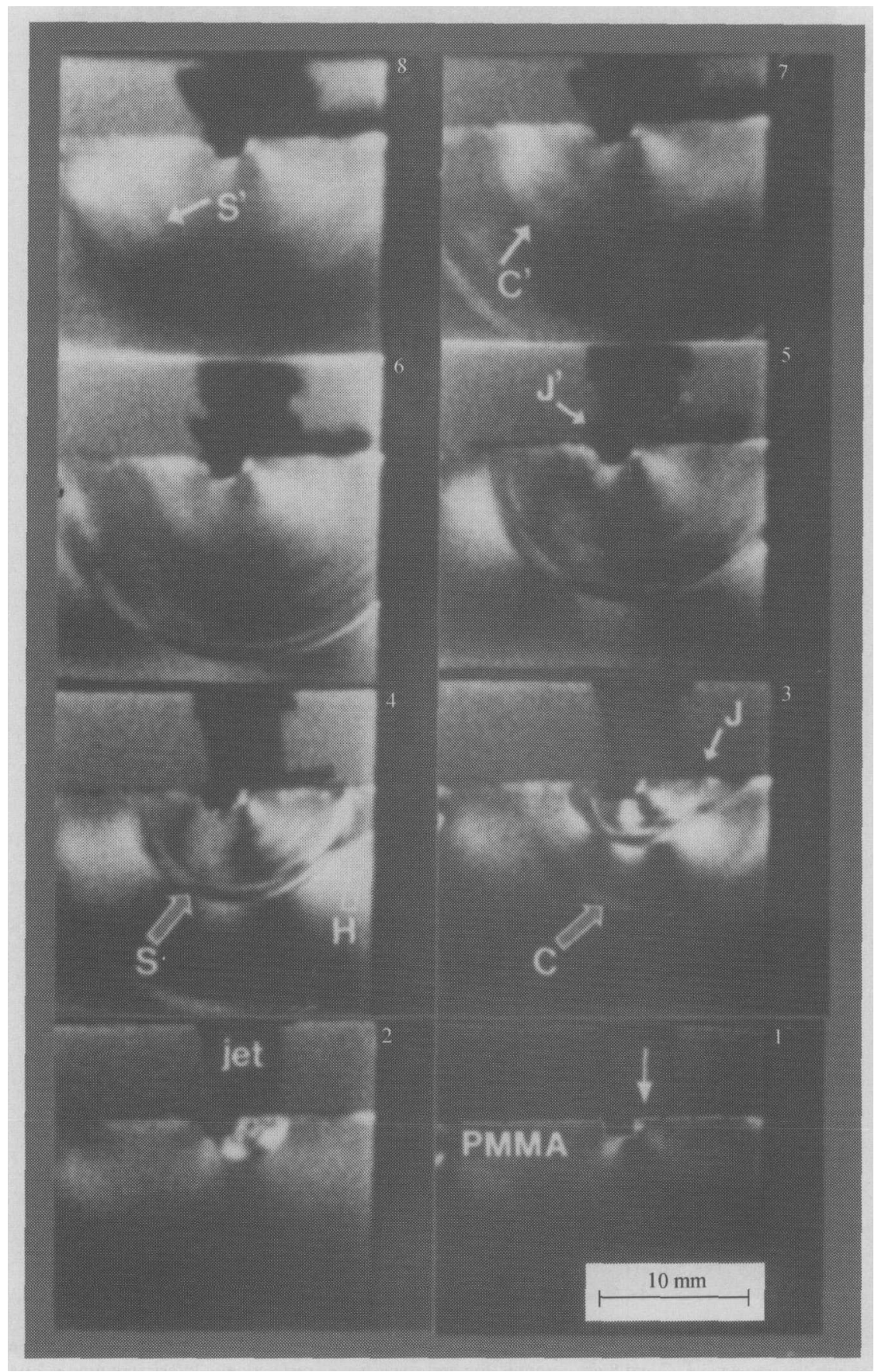

Fig. 6. High speed photographs of a $450 \mathrm{~m} / \mathrm{s}$ water jet impact on a PMMA notch. The white arrow in frame 1 shows the impact site. C, S and $\mathrm{H}$ are compression, shear and head waves respectively; $\mathrm{C}^{\prime}$ and $\mathrm{S}^{\prime}$ are secondary compression and shear waves respectively; $\mathrm{J}$ is side jetting; $\mathrm{J}^{\prime}$ is rebounding jetting. Interframe time $=2 \mu \mathrm{s}$.

Copyright by Science in China Press 2004 
$\mathrm{V}$ shape groove. The impact pressure on an inclined surface is less than that on a horizontal surface and the motion of the jet on the inclined surfaces exerts a strong shear stress to the solid ${ }^{[9,10]}$.

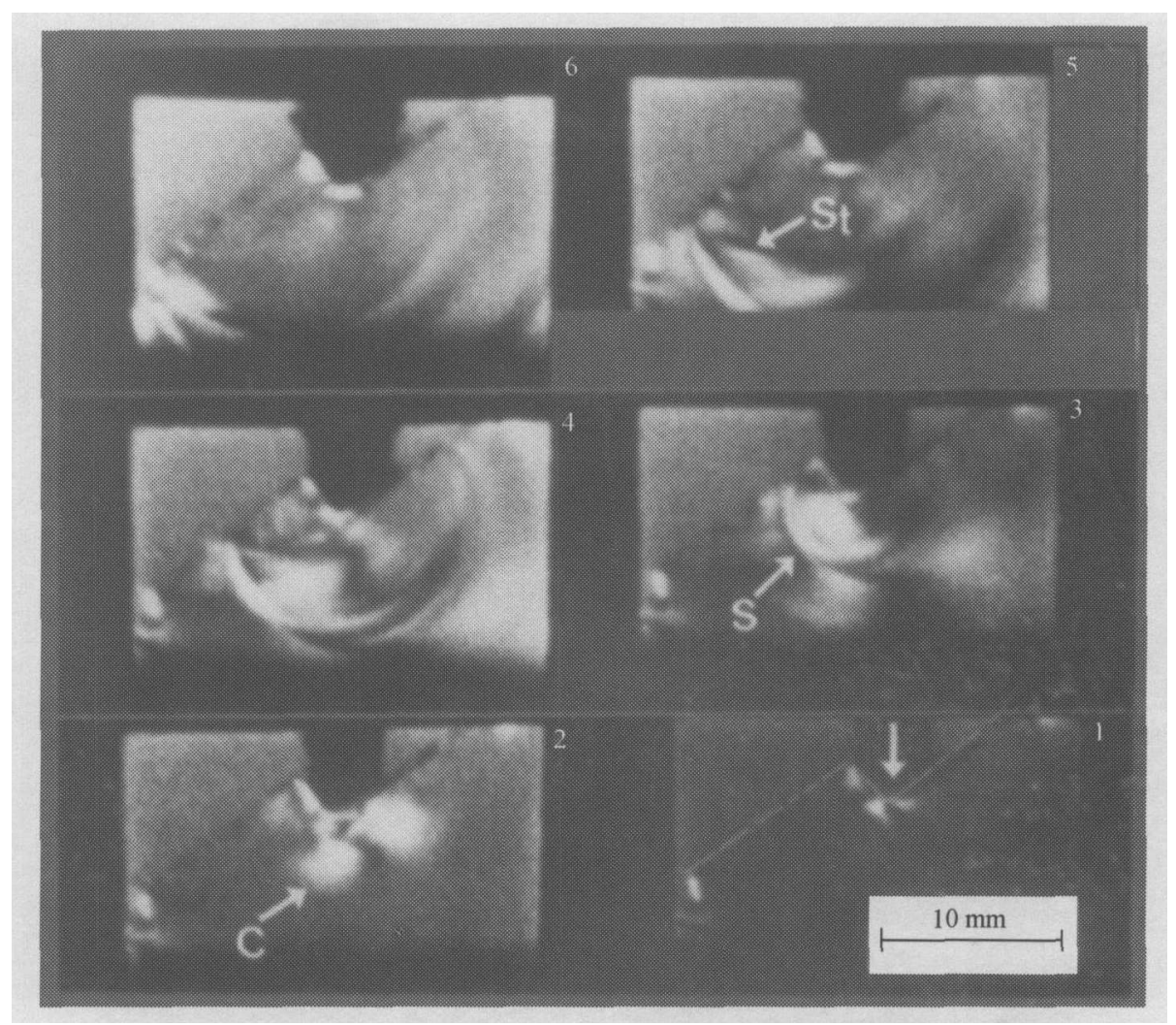

Fig. 7. High speed photographs of a $450 \mathrm{~m} / \mathrm{s}$ water jet impact on a V shape groove. The white arrow in frame 1 shows the impact site. $\mathrm{C}$ and $\mathrm{S}$ are compression and shear waves respectively; $\mathrm{S}_{\mathrm{t}}$ is the reflected transverse wave of $\mathrm{S}$ from the left free surface. Interframe time $=2 \mu \mathrm{s}$.

\subsection{Impact damage of material by side jetting}

To test the erosion potential of the side jetting, an experiment shown in fig. 8(a) was designed. An $850 \mathrm{~m} / \mathrm{s}$ water jet impacts on a steel block. A $3 \mathrm{~mm}$ thick PMMA plate is put on the right side of the steel block, which is facing the side jetting. The velocity of the side jetting is predicted at $\sim 2 \mathrm{~km} / \mathrm{s}^{[3]}$. Fig. 8 (b) is the photograph of the eroded PMMA plate surface. Fig. 8(c) is an enlargement of fig. 8(b). It is seen from figs. 8(b) and 8(c) that there exist two types of material damage: (1) material spalling due to the liquid swilling out; (2) Rayleigh surface wave interacts with the pre-existed flaws in the material so that short discrete cracks appear on the solid surface ${ }^{[2]}$.

It must be indicated that the shape of the side jetting head is usually irregular, which is very thin and may consist of fine droplets. Therefore, even though the side jetting head is in high speed, it may not have sufficient erosion potential. The high speed 


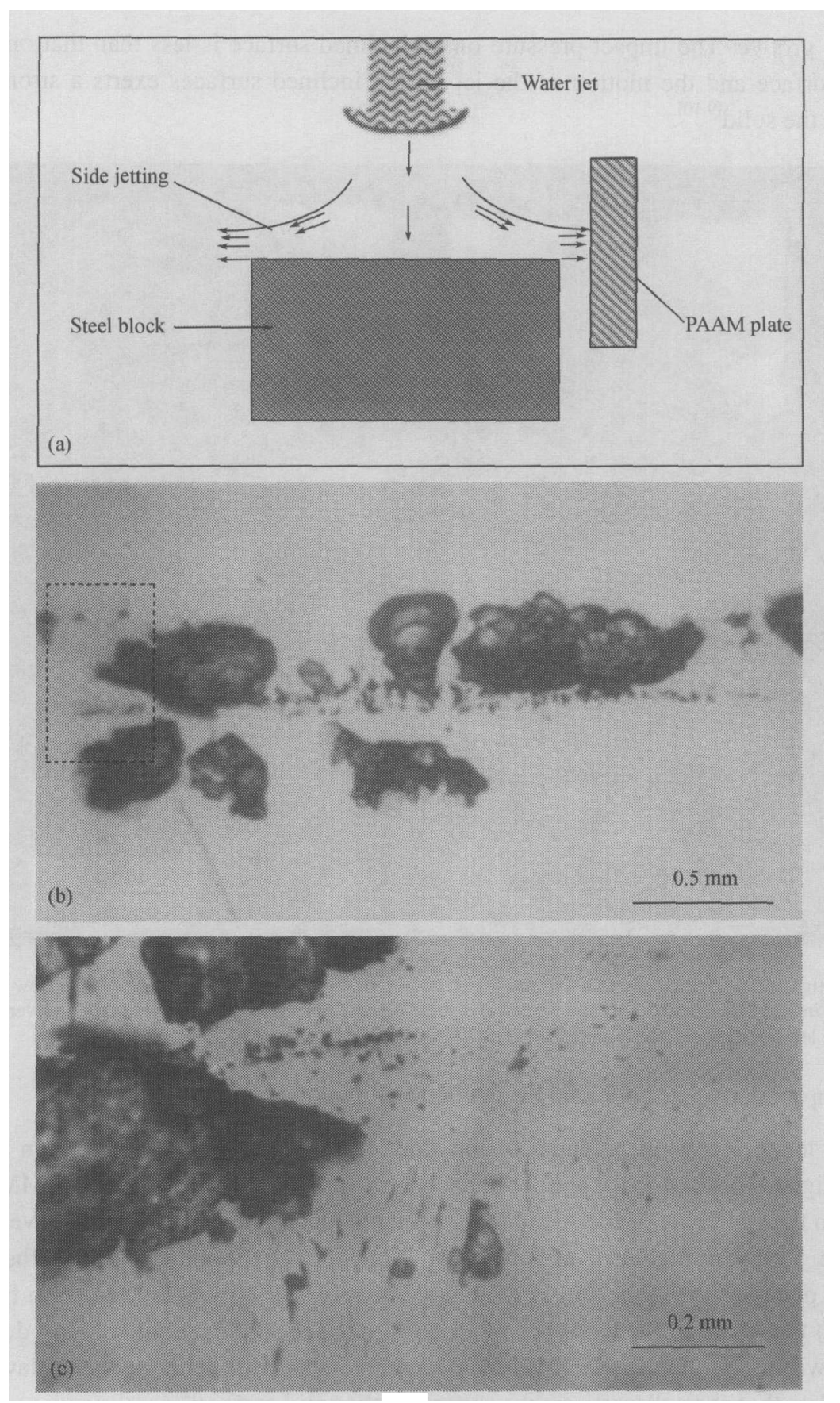

Fig. 8. Impact damage of $3 \mathrm{~mm}$ thick PMMA plate by the high speed side jetting. Water jet of $850 \mathrm{~m} / \mathrm{s}$ from a $0.8 \mathrm{~mm}$ nozzle impacts on a steel block. The gas between the PMMA plate and the steel block is $1 \mathrm{~mm}$. (c) is the enlargement of (b), and the square broken line in (b) shows the enlarged position.

photographs of fig. 9 confirm this. After a $450 \mathrm{~m} / \mathrm{s}$ water jet impacts on the PMMA block, the left side jetting crosses a $1.5 \mathrm{~mm}$ wide silt gap to impact on the opposite solid

Copyright by Science in China Press 2004 


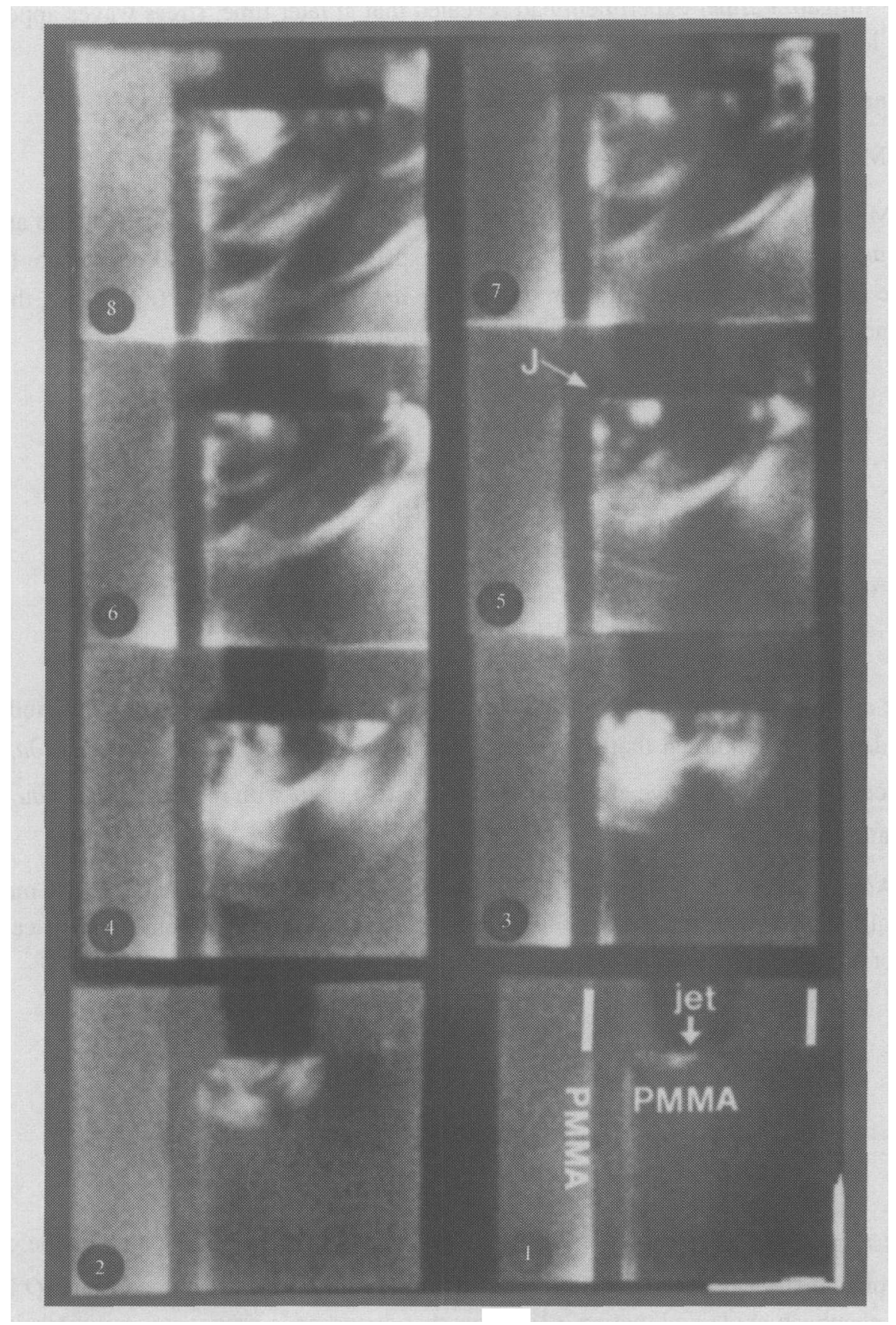

Fig. 9. After the impact of a $450 \mathrm{~m} / \mathrm{s}$ water jet on a PMMA block, the side jetting $\mathrm{J}$ is across the $1.5 \mathrm{~mm}$ wide slit to impact on the left solid wall. Interframe time $=1 \mu \mathrm{s}$.

wall. At the time of fig. 9(5), the side jetting $J$ has just left the solid edge which is on the right of the slit gap. At the time of fig. 9(6), the side jetting has arrived at the opposite wall. However, in fig. 9(7) and 9(8), no stress waves appear in the solid that is on the left 
of the slit gap. Further experiment had revealed that at later time, stress waves appeared in the left solid.

\section{Conclusions and discussion}

\subsection{Mechanism of stress waves generation}

Miller and Pursey ${ }^{[14]}$ deduced theoretical expressions of stress waves due to an oscillating normal point loading $Q e^{i \omega t}$ on a half space. Taking spherical coordinate $(r, \theta$, $\phi$ ), the radius of the loading area on the surface $a \rightarrow 0$, in the far field ( $r$ is large), the radial and transverse components of the displacement $u$ are

$$
\begin{aligned}
& u_{r} \sim-\frac{a^{2} Q}{2 c_{44}} \frac{e^{i\left(\omega t-k_{1} r\right)}}{r} \Theta_{1}(\theta), \\
& u_{\theta} \sim-\frac{i a^{2} k^{3} Q}{2 c_{44}} \frac{e^{i\left(\omega t-k_{2} r\right)}}{r} \Theta_{2}(\theta), \\
& k_{1}=\omega\left(\rho / c_{11}\right)^{1 / 2}, k_{2}=\omega\left(\rho / c_{44}\right)^{1 / 2}, \\
& k=\left(c_{11} / c_{44}\right)^{1 / 2}=k_{2} / k_{1}=\{2(1-v) /(1-2 v)\}^{1 / 2},
\end{aligned}
$$

where $c_{11}$ and $c_{44}$ are compression and shear elastic constants, $v$ is Poisson ratio, and $\rho$ is solid density. It is known that the partial derivative of $u_{r}$ with respect to time $\partial u_{r} / \partial t$ produces compression wave and the partial derivative of $u_{\theta}$ with respect to time $\partial u_{\theta} / \partial t$ generates shear wave.

Achenbach (page 301 in ref. [13]) introduced a rigorous and more direct mathematical formula of stress waves. For a transient line loading on a solid half space, the horizontal displacement is

$$
\begin{aligned}
\frac{2 \pi \mu s_{T}{ }^{2}}{Q} u(x, y, t)= & \frac{x y}{r^{2}}\left[\frac{2}{r^{2}}\left(t^{2}-s_{L}{ }^{2} r^{2}\right)^{1 / 2}+\frac{s_{L}{ }^{2}}{\left(t^{2}-s_{L}{ }^{2} r^{2}\right)^{1 / 2}}\right] H\left(t-s_{L} r\right) \\
& -\frac{x y}{r^{2}}\left[\frac{2}{r^{2}}\left(t^{2}-s_{T}{ }^{2} r^{2}\right)^{1 / 2}+\frac{s_{T}{ }^{2}}{\left(t^{2}-s_{T}{ }^{2} r^{2}\right)^{1 / 2}}\right] H\left(t-s_{T} r\right),
\end{aligned}
$$

where $r^{2}=x^{2}+y^{2}, s_{L}=1 / c_{L}, s_{T}=1 / c_{T}, c_{L}$ and $c_{T}$ are the velocities of the compression and shear waves, $H$ is Heaviside step function, $\mu$ shear elastic constant, $Q$ loading. Achenbach explained that eq. (2) shows that disturbance propagates outwards in the velocities of $c_{L}$ and $c_{T}$, and the waves fronts are the semi-circle with radius of $c_{L} t$ and $c_{T} t$ from the loading point. The mechanisms of generation of head and surface waves were explained in detail in ref. [13]. 


\subsection{Impact pressure}

When a high speed liquid drop (or jet) impacts on a rigid solid surface, the pressure at the impact center is $P=\rho C V$, where $\rho, C$ and $V$ are liquid density, liquid shock wave velocity and impact velocity. If the solid is elastic, the formula of the impact pressure needs to be modified by considering the elasticity ${ }^{[3]}$. When a $450 \mathrm{~m} / \mathrm{s}$ water jet from a $0.8 \mathrm{~mm}$ diameter nozzle impacts on a PMMA surface, the central impact pressure is 0.5 $\mathrm{GPa}$, and its duration is $0.5 \mu \mathrm{s}^{[10]}$. Because of this strong loading, various stress waves in the solid become visible. It also means that the high speed liquid impact has sufficient damaging potential.

It has been confirmed in theory that the maximum impact pressure does not appear at the impact center but appears at the contact edge of liquid/solid ${ }^{[2,3,5,10]}$. Now the most commonly used formula is that, according to the experiment by Rochester and Brun$\operatorname{ton}^{[15]}$, the maximum impact pressure is three times the water hammer pressure, that is, $P_{e}=3 \rho \mathrm{CV}$. However, the experiment done by Rochester and Brunton has never been repeated by other people. This may remind us that the problem is still open.

\subsection{Side jetting and others}

Using high speed photography, the complicated stress waves propagation phenomena such as reflection, interference and diffraction during a high speed liquid jet impact on a corner, step change, notch and groove were observed. It was found that the waves propagation behaviors depend not only on the surface geometry of materials but also on the fluid dynamics in liquid/solid contact. Although the elastic waves theory has been a powerful tool ${ }^{[12,13]}$ and Graff $^{[8]}$ and Harker ${ }^{[11]}$ also provided experimental data, concerning the problem discussed in this paper, there have been no satisfactory answers in open literature. In calculating material stress and strain states under the impact of high speed liquid using commercial codes, it is necessary to know the propagation of stress waves in time and spatial spaces. This paper can provide such a reference.

The experiment showed that the side jetting is in high speed and its impact on a surface discontinuity can easily cause material damage. The head part of a side jetting may consist of fine droplets so that it may not have erosion potential. It must be noted that the jet tip is followed by larger liquid that is in high speed as well as is damaging.

This experiment confirmed the compression, shear, head and surface waves predicted by the classical elastic waves theory. The observed wave reflection process was in agreement with the holographic visualization of spherical wave reflection by Shi et al. ${ }^{[16]}$. It is known from plastic wave theory ${ }^{[12]}$ that the velocity of plastic wave is less than that of elastic wave. This kind of wave was found in the experiment. Even though a plastic wave is generated, it will be dissipated after leaving the impact point for a distance. The mechanism of the generation of a plastic wave concerns material surface hardness, loading rate, dynamic yielding stress, etc., which is beyond the scope of this paper. 
Acknowledgements This project was partly supported by a Tang Zhao-Qian Scholarship of Xi'an Jiaotong University and the "BaiRen" Plan of the Chinese Academy of Sciences.

\section{References}

1. Momber, A. W., Energy transfer during the mixing of air and solid particles into a high-speed waterjet: an impact-force study, Experimental Thermal and Fluid Science, 2001, 25: 31—41.

2. Kennedy, C. F., Field, J. E., Damage threshold velocities for liquid impact, J. Mater. Sci., 2000, 35: 53315339.

3. Shi, H. H., Theoretical and experimental investigations on the rotor blading erosion by the impact of water drops in wet steam turbines, PhD Thesis (in Chinese), Xi'an Jiaotong University, Xi'an, 1989.

4. Zhang, Z. C. (ed.), Hypersonic Aerodynamic Heating and Thermal Protection (in Chinese), Beijing: Defence Industry Press, 2003.

5. Zhang, D., Xie, Y. H., Study on nonlinear coupling wave model for liquid drop-solid impact, Chinese Journal of Aeronautics, 2002, 15(4): 222-227.

6. Shi, H. H., Perforation of metal plates under high-speed and high-viscosity liquid jet impacts, Explosion and Shock Waves (in Chinese), 2003, 23(3): 193-199.

7. Shi, H. H., Takagi, K., Erosion of aluminum plates by the impact of high-speed polymer solution jets, Proc. 7th Natl. Conf. on Explosion Mechanics, Kunming, November 7-13, 2003; Explosion and Shock Waves (in Chinese), 2003, 23(Suppl): 273-274.

8. Graff, K. F., Wave Motion in Elastic Solids, Oxford: Clarendon Press, 1975.

9. Shi, H. H., Dear, J. P., Oblique high-speed liquid-solid impact, JSME Int. J. Ser. I, 1993, 11: 79—93.

10. Shi, H. H., Field, J. E., Pickles, C. S. J., High-speed liquid impact onto the solid targets with complex surface geometry, in Proc. 21st Int. Symp. on Shock Waves, Paper 5190, Great Keppel Island, Australia, July 20-25, 1997, 1229-1233.

11. Harker, A. H., Elastic Waves in Solids, Adam Hilger: IOP Publishing Ltd, 1988.

12. Kolsky, H., Stress Waves in Solids, Oxford: Clarendon Press, 1953.

13. Achenbach, J. D., Wave Propagation in Elastic Solids, Amsterdam: North-Holland Publishing Company, 1973.

14. Miller, G. F., Pursey, H., On the partition of energy between elastic waves in a semi-infinite solid, Proc. Roy. Soc. London Ser. A, 1955, 233: 55-69.

15. Rochester, M. C., Brunton, J. H., Pressure distribution during drop impact, in Proc. 5th Int. Conf. on Erosion by Liquid and Solid Impact (ed. Field, J. E.), Cambridge, UK: Cavendish Laboratory, 1979, paper 6.

16. Shi, H. H., Takayama, K., Nagayasu, N., The measurement of impact pressure and solid surface response in liquid-solid impact up to hypersonic range, Wear, 1995, 186-187: 352-359.

Copyright by Science in China Press 2004 